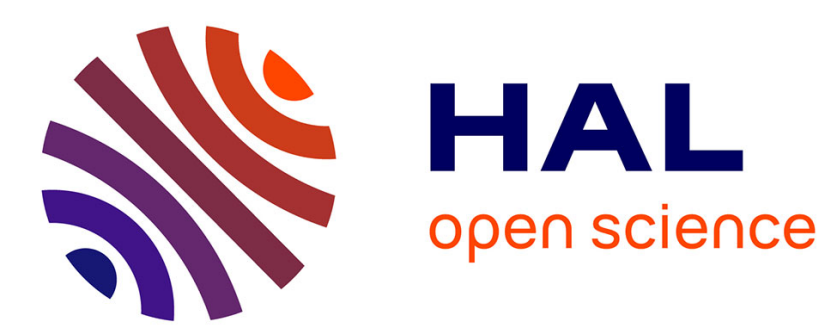

\title{
Elementary Stability Considerations for Bowed-String Motion
}

\author{
Gabriel Weinreich, René Causse
}

\section{To cite this version:}

Gabriel Weinreich, René Causse. Elementary Stability Considerations for Bowed-String Motion. Journal of the Acoustical Society of America, 1991, 2 (89), pp.887-895. hal-01105555

\section{HAL Id: hal-01105555 \\ https://hal.science/hal-01105555}

Submitted on 20 Jan 2015

HAL is a multi-disciplinary open access archive for the deposit and dissemination of scientific research documents, whether they are published or not. The documents may come from teaching and research institutions in France or abroad, or from public or private research centers.
L'archive ouverte pluridisciplinaire HAL, est destinée au dépôt et à la diffusion de documents scientifiques de niveau recherche, publiés ou non, émanant des établissements d'enseignement et de recherche français ou étrangers, des laboratoires publics ou privés. 


\title{
Elementary Stability Considerations for Bowed- String Motion
}

\author{
Gabriel Weinreich, Rene Caussé \\ J. Acoust Soc. Am. 89 (2), February 1991 \\ Copyright (C) ASA 1991
}

( Received 9 March 1990; accepted for publication 17 October 1990 )

Using the approach first pioneered by Raman, the Helmholtz motion of a bowed string is discussed as a special case of "two-velocity motions," in which a given point (at which the bow is located ) alternates, in the course of a cycle, between two constant velocities. The fact that the bow typically presents a negative resistance to the string during the "slipping" part of the cycle is adduced as a reason for the "duty cycle," that is, the fraction of the period that corresponds to slipping, to try to become as short as possible. It is shown that, for a string without dissipation or stiffness, this duty cycle can be arbitrarily low for general bow positions; data obtained with the "digital bow" illustrate this behavior. It is shown theoretically, and confirmed with computer simulations, that instabilities arising from the negative slipping resistance cannot be eliminated by assigning a finite positive value to the sticking resistance. The apparent stability of Helmholtz motion observed in real playing situations remains a puzzle.

\section{Introduction}

The "Helmholtz motion" of a string is one in which the string consists, at any instant of time, of two straight-line segments, joined by a constant slope discontinuity or "kink" that moves at the speed of transverse waves from one end of the string to the other and returns with opposite sign. At any given location, the string velocity alternates between two constant values that are opposite in sign and whose ratio is equal to the ratio of distances from the point in question to each of the string terminations. This type of motion was first observed experimentally, and discussed theoretically, by Helmholtz. [1]

The fact that at each location on the string, including an imagined "bowing point," the Helmholtz motion corresponds to an alternation between two constant string velocities makes it easy to conceptualize it as an embodiment of the kind of"stick-slip" phenomenon that we associate with the dry friction between string and rosined bow. In particular, one usually thinks of the interval of smaller velocity (which lasts for a longer time) as "the string sticking to the bow;" the larger, oppositely directed velocity then corresponds to "the string slipping back," only to be "caught" by the bow once again.

Considerable advances have added to our understanding of bowed-string motion in recent years; among them, computer simulations ${ }^{[3]-[4]}$ have shown how, under various realistic conditions, Helmholtz motion, or some close approximation to it, indeed arises when a string that is originally at rest has a bow applied to it. In fact, such simulations have provided considerable fine detail on likely realistic regimes of motion. Yet, for some purposes, such approaches give too much information in that the elementry "stickslip" concept is not always easily applied to their understanding. Our purpose in this paper is to develop a somewhat different conceptual approach, which, in our opinion, can do much to clarify the discussion of why Helmholtz motion is to be expected and under what circumstances it is stable. The point of view we use has some overlap with older work of Raman, $\stackrel{[5]}{[}$ but is developed considerably further.

\section{The Ideal Model}


We begin by reviewing briefly the well-known simple model of the bowed string as presented, for example, by Cremer. ${ }^{[2]}$ For this purpose, we assume, first, that the string is linear, dispersionless, dissipationless, and terminated by absolutely rigid supports; second, that the frictional characteristic between the bow and the string can be specified as a curve that gives the frictional force as a function of relative velocity at that instant. The same curve then specifies the frictional force as a function of absolute string velocity if it is offset horizontally by an amount equal to the steady bow speed ( Fig. I ) .

Our frictional characteristic is drawn so that its "sticking" portion is not vertical but has a finite slope; that is, we do not assume that "sticking" automatically implies that the relative velocity between string and bow hair is identically zero. In fact, an infinite slope is not required for further development of the theory, but can always be inserted later as a special case. Specifically, "the digital bow" (which supplied some experimental data to be quoted below) has an adjusta- ble frictional characteristic whose slope is ordinarily not infinite.

Physically, on the other hand, the concept of"sticking" does imply that the force-velocity characteristic has an infinite slope

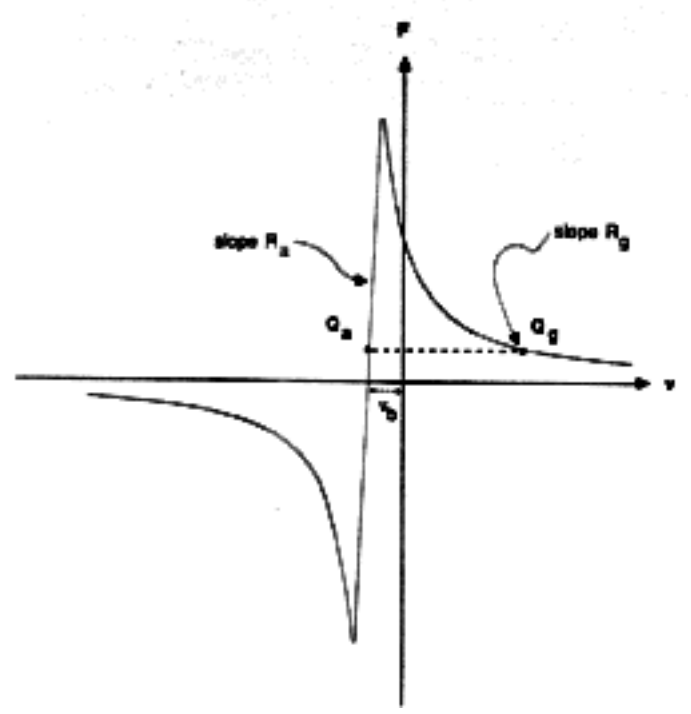

FIG. I. Generic frictional characteristic, showing the (negative of) the force exerted by the bow on the string as a function of the string's velocity $\mathrm{Q}_{\mathrm{a}} \mathrm{a}$ and $\mathrm{Q}_{\mathrm{g}}$ are the point corresponding lo sticking ("adhérence")and slipping ("glissage"). The line $\mathrm{Q}_{\mathrm{a}} \mathrm{Q}_{\mathrm{g}}$ is horiontal and is divided by the force axis in the ratio $\beta /(1-\beta)$ for Helmholtz motion or, in the case of a general twovelocity motion, in the ratio $\gamma /(1-\gamma) ; \mathrm{R}_{\mathrm{a}}$ and $\mathrm{R}_{\mathrm{g}}$ are the two "resistances" that is, the slope at the operating points.

Thus, for eample. it was pointed out by McIntyre ${ }^{[6]}$ that one can use the bow to pull the string to one side and hold it there for a practically indefinite length of time. Yet, there are a number of factors that. in practice, may produce an "effective slope" which is not infinite. One is the torsional motion of the string; $77-[8]$ another is the dynamical vibration of the bow system itself, which may make the instantaneous velocity of the bow hair deviate from the nominally constant velocity of an idealized player's wrist. In the latter connection, Cremer ${ }^{[8]}$ argues that effects due to longitudinal (stretching) vibration of the bow hair themselves are probably negligible; nonetheless, other motions of the bow system, such as bending of the stick, cannot be as easily ruled out.

One should note that the assumption of a dispersionless string is equivalent to the absence of any stiffness, that is, to any contribution to the potential energy that depends on the curvature of the string. Without such an assumption, any configuration that contains a kink would imply an infinite potential energy and would thus be ruled out immediately. 
Under these assumptions. the Helmholtz motion (as defined in the first paragraph of this paper) is a possible solution of the free string equation, that is, a possible motion of the string without a bow $[1]-[5]-[2]$. Having established this fact. we must ask whether, and how, it is also a solution of the equations that govern the system when the bow is applied.

Let $L$ be the total length of the string, and $\beta_{L} L$ and $(1-\beta L)$ the distances from the bowing point to either string end. Since the Helmholtz motion requires the string at the bowing point to move with one constant velocity for a fraction $\beta$ of a period, then with a different (but again constant) velocity for the remaining 1$\beta$ of a period; and since we have assumed a frictional characteristic that unequivocally specifies the force as a function of the velocity; it follows that the bow will exert one constant force on the string for a fraction $\beta$ and another constant force for the remaining fraction $1-\beta$ of the period. In fact, these two constant forces must be equal to each other; otherwise, the bow would do a net amount of work on the string in the course of a period, which is impossible if the motion is steady and there is no dissipation. On this basis, we can say that the two points marked $\mathrm{Q}_{\mathrm{a}}$ and $\mathrm{Q}_{\mathrm{g}}$ in Fig. 1, which characterize the two states normally called "sticking" and "slipping," must lie on the same horizontal line. (Since "sticking" and "slipping" have the same initial letter, we use the subscripts a and g derived from the corresponding French words "adhérence" and "glissement." )

At the same time, we know that the two velocities of the string at the bowing point must have magnitudes inversely proportional to the time spent in the respective states, so as to make the net displacement in the course of a complete period vanish. For Helmholtz motion, this means that the two velocities must be in the ratio $-\beta /(1-\beta)$. Given the frictional characteristic and the speed and location of the bow, the two requirements that $\mathrm{Q}_{\mathrm{a}}$ and $\mathrm{Q}_{\mathrm{g}}$ lie on the same horizontal line, and have abscissae whose ratio is as given above, in most cases determine the location of these two points.

We ask next whether the Helmholtz motion specified by this construction is dynamically possible; for, although it is known to be a solution of the free string equation, the string is now no longer free because the bow is applying a force to it. Since, however, this force is constant in time. its effects can be taken care of by superposing upon the Helmholtz motion a stationary configuration consisting of two straight line segments with a fixed kink at the bow location, and having a slope discontinuity corresponding to the static displacement of the same string under the same constant localized transverse force. Because linearity of the string has been assumed, the superposition of this stationary kink upon the moving Helmholtz kink now constitutes a solution for a string which is free except for the application of the (time-independent ) bow force ${ }^{[9]}$.

\section{Other two-velocity Motions}

It should be noted that the foregoing argument, which establishes the Helmholtz motion (plus a stationary kink ) as a possible solution of the ideal string with a bow applied at location ${ }^{\beta} L$, works equally well for any other motion of the free string which exhibits, at the bow location $\beta L$, one constant velocity for some fraction $y$ of a period and some other constant velocity for the remaining fraction $I-\gamma$. It is not necessary that $\gamma$ be equal to $\beta$, as is true for the Helmholtz motion itself. Of course, the construction that determined the bow speed to which such a motion corresponds would be somewhat different. requiring that the force axis divide the line segment $\mathrm{Q}_{\mathrm{a}} \mathrm{Q}_{\mathrm{g}}$ (Fig. 1) in the ratio $\gamma /(1-\gamma)$ rather than $\beta /(1-\beta)$.

Indeed, as was already shown by Raman, $\frac{[5]}{5}$ there do exist other possible motions of a free string for which the velocity at a given location alternates between two fixed values (although the Helmholtz motion is the only one for which such a statement can be made about every point of the string). To clarify this, we ask the following question: Given an arbitrary function of time, under what circumstances can it correspond to the velocity of a given point of the string when it is moving freely

We know, of course, that any vibration of the free string can be expanded in normal modes; and since we have assumed that there is no dispersion and that the endpoints are fixed, the period of the $n$th normal 
mode will be $\tau / \mathrm{n}$, where $\tau$. is the string's fundamental period. Clearly, any superposition of such modes will still have an overall periodicity $\tau$, from which it follows that a function which purports to represent the time dependence of velocity of a given point must itself have that periodicity. This is an obvious necessary condition.

It this condition is satisfied, we can expand the given function as a Fourier series; we then interpret each coefficient as the strength of the corresponding normal mode at the given string position. In this way an arbitrary periodic function can be seen as all expansion in the normal modes, and hence a possible free motion of the string, except for one restriction: If the given point is an exact node of some normal mode, then the corresponding Fourier component cannot be represented in the velocity function. More specifically, if the point in question lies at a fraction $\mathrm{m} / \mathrm{P}$ of the string length (where $\mathrm{m}$ and $\mathrm{P}$ are integers), the harmonics P, 2P, etc., must be absent from the velocity as observed at that point. (One may ad that under any circumstances, the "zeroth harmonie" must be absent from the velocity to prevent any secular drift of the string at least so long as the terminations are fixed.) With this understanding, we now ask what the possible free string motions are for which the velocity at location $\beta_{L}$ alternates between two fixed values.

Let $\gamma$, as above, be the "duty cycle" of a proposed two-velocity motion, that is, the fraction of a period spend in one of the two states. [Again, to preclude a secular drift, the two-velocities must have opposite signs, and their magnitudes must be in the ratio $(\mathrm{I}-\mathrm{y}) / \mathrm{y})$.] We then see from the foregoing discussion that, if $\beta$ is not rational, any duty cycle can be constructed, but, for $\gamma$ rational, this is not the case. We show in Appendix A that, if $\gamma=\mathrm{m} / \mathrm{P}$, where $\mathrm{m}$ and $\mathrm{P}$ are integers and the fraction is in its lowest terms, the possible values of $\gamma$ are limited to integer multiples of $1 / P$. In particular, the shortest duty cycle of a free string vibration which is a two-velocity motion at $\beta=\mathrm{m} / \mathrm{P}$ is $\gamma=\mathrm{I} / \mathrm{P}$.

In physical cases, of course, the qualitative distinction between rational and irrational values of $\beta$ cannot be meaningful. Instead, we would expect the behavior of the string to differ between a regime of "small P" and one of "largeP," the comparison being with some "critical P" determined, perhaps, by the rounding of the Helmholtz kink due to string stiffness or bow width.

\section{The "Staircase" Function}

The discussion of the previous section makes it clear that the velocity function at $\beta=\mathrm{m} / \mathrm{P}$ must lack the harmonics of order $\mathrm{P}, 2 \mathrm{P}$, etc. This does not in itself mean, of course, that the corresponding normal modes are necessarily absent from the string; if the dissipation is zero, as we have assumed, their amplitudes remain indeterminate. It is clear however that a bow of zero width placed at a certain location is not capable of feeding any energy into modes which have nodes at that point. Therefore, if some dissipation does exist-even if it is small one may expect those modes to decay with time, so that they would not be represented in the steady-state motion.

If harmonics of order P, 2P, etc., are removed, the shape of the force exerted by the string on the bridge ( which can be thought of as the "signal" sent by the string to the violin body) will be qualitatively modified: The displacement very near the bridge will change from a "sawtooth" to a "staircase," in which the uniform ramp of the sawtooth is replaced by $\mathrm{P}$ horizontal "steps." $[10]-11$ It is true, of course, that if $\mathrm{P}$ is fairly high (as it would have to be for a bow at a normal playing position rather near the bridge), the quantitative modification may not be very large. Nonetheless, there is something unphysical about a phenomenon that seems to exist when $\beta$ is rational but disappears abruptly when it is irrational. As we shall see the existence of other two-velocity motions will allow us to reformulate the phenomenon so as to make its dependence upon $\beta$ more continuous.

\section{Stability}

Having shown that any two-velocity motion is a possible motion of the bowed string (provided that an 
appropriate stationary kink is included and that $\beta$ is not rational), we now imagine that such a motion has been established, and ask whether it is stable; in other words, we ask what would happen ifa small perturbation were superimposed on it. The linearity of the system other than at the bow will allow the two to coexist without interacting; at the bow, however, the perturbation will cause additional forces determined by the slopes of the frictional characteristic at the two "operating points" $\mathrm{Q}_{\mathrm{a}}$ and $\mathrm{Q}_{\mathrm{g}}$ in Fig. 1 which correspond to nominal "sticking" and "slipping." It is clear from the general nature of the characteristic that the sticking point will have a positive slope, corresponding to a "positive resistance" that drains energy from the perturbation, whereas the slipping point will have a "negative resistance" that feeds energy into it. These effects will, of course, be most pronounced when the magnitude of the resistance is comparable to the characteristic impedance of the string. In this way, the stability of any given small perturbation over the course of a complete cycle can be discussed. One immediate corollary is that, if the "sticking" slope is infinite then the Helmholtz motion Is unconditionally unstable. This fact was already obtained by Friedlander using a completely different approach $\frac{12}{2}$ and in- terpreted by Mclntyre, Schumacher, and Woodhouse in terms of subharmonic generation. $\underline{13}$

With finite slopes, the question of stability requires a more detailed calculation, which does not become trivial no matter what the values of the slopes. Consider, for example, the (not particularly realistic) case in which both slopes are

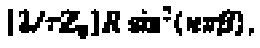

from which one might be led to conclude that, if the string spends a fraction $\gamma$ of its period with a resistance $\mathrm{R}_{\mathrm{g}}$ attached, and the remaining fraction $1-\gamma$ with a resistance $\mathrm{R}^{\mathrm{g}}$, the net rate of exponential decay of the amplitude of a perturbation corresponding to the nth normal mode will be

$\left[2 /+Z_{1}\right]\left[R_{1} \gamma+R_{-}(1-\eta)\right] \operatorname{in}^{2}(n=f)$.

(2)

In this expression, $\mathrm{R}_{\mathrm{a}}$ and $\mathrm{R}_{\mathrm{g}}$ are, respectively, the sticking slope (which is positive) and the slipping slope (which is negative); $Z_{0}$ is the characteristic impedance of the string; $\beta$

is the (fractional) location of the bowing point; and $\gamma$ is the dutycycle. (For a Helmholtz motion, $\gamma$ and $\beta$ are, of course, equal.) This would lead us to formulate as a stability criterion for all modes.

In fact, as we shall see in more detail in Sec. VII, this criterion is by no means sufficient. The fallacy is that it is really necessary to define the "modes during sticking" separately from the "modes during slipping" and to reexpand the perturbation first in one set then in the other, twice during each cycle. The coherent behavior of these successive reexpansions can then cause instabilities whose overall periodicity is nowhere near any of the normal modes, as in the well-established subharmonic generation discussed by Mc-lntyre el al. $\frac{13}{1}$ It is also possible as we shall see in Sec. VII, to construct perturbations that do have the correct fundamen- tal frequency, but that are so distributed over the string as to be essentially absent from the bow location during the sticking part of the cycle. We refer to them as "between-the-rain- drops" motions, drawing on the metaphor of a person remaining dry in the rain by "running between the raindrops." Under those circumstances, the growth due to the (nega- tive) slipping resistance is not compensated by any decay due to the (positive) sticking resistance, making the perturbation considerably more unstable than the criterion of Eq. (3) would suggest. Appendix B demonstrates how motions of this type may be constructed.

Nonetheless, the qualitative idea expressed by Eq. (2) that a relatively short duty cycle (that is, a short slipping time) enhances the stability of a two-velocity motion generally remains valid. Such a condition corresponds to the statement made by Cremer, $\frac{14}{}$ that "the bow tries always to maximize the sticking time," even though the explanation given by him in terms of a simple friction-driven oscillator seems to us to be insufficient. 
The following specific consequence of such a condition is worth noting immediately. There is, in the common description of Helmholtz motion (or of any other two-velocity motion), an ambiguity concerning the duty cycle in that it is assumed that, of the two fractions $\gamma$ and 1- $\gamma$ (which, for Helmholtz motion, are the same as, and $\beta_{1}{ }^{\beta}$ ), the shorter one always corresponds to slipping and the longer one to sticking. Kinematically, however, an "inverted" Helmholtz motion is equally possible. If, for example, the bow is placed at a point that lies at $10 \%$ of the string length, one can construct a Helmholtz motion corresponding to it which slips for $90 \%$ of the cycle and sticks for $10 \%$, with the bow speed corresponding to the larger velocity. Our discussion makes it clear, however, that such an inverted motion (which, to our knowledge. has never been observed) would be very much more likely to run into stability problems.

\section{Connection with Experiment}

Whatever the merits of the "ideal model" that we described in Sec. I, it is surely not an exact one, so that comparison with experiment must proceed with some care. Now it is commonly stated that a bowed string in steady vibration does exhibit Helmholtz motion; nonetheless, the observed transition between sticking and slipping is not instanta- neous, giving rise to the phenomenon of "rabbit ears" in the force. $\underline{15-7}$ Since the ideal model calls for a force that is absolutely constant, and dissipation that is absolutely zero, relatively small deviations may give rise to important modifica- tions of the theory.

Specifically. the clear theoretical proof that ideal Helmholtz motion with perfect sticking is always unstable leads us to ask which specific deviation from the model produces the stability that is experimentally observed: Is it the fact that the sticking is. in effect, not perfect (due perhaps to torsion ${ }^{7-1} \underline{13}$ or other effects ), or the fact that the motion deviates kinematically from Helmholtz motion? It should be added that the presence of weak dissipation (which is surely there in a real case) cannot be simply taken care of by slightly raising the point $\mathrm{Q}_{\mathrm{a}}$ relative to $\mathrm{Q}_{\mathrm{g}}$ in Fig. 1 . True, that would provide a net energy influx from the bow, but the force would no longer be constant in time, so that the Helmholtz motion plus a fixed kink would no longer be a solution of the string equations.

It is interesting, in this connection, to present some relevant data from our experiments with the "digital bow." In this setup, a real string is driven, not by a physical bow, but by an electronic system that substitutes for it by electrodyna- mically exerting a force that is determined by the string's instantaneous velocity at the imagined "bowing point." Specifically, the string's velocity is measured photoelectrically and the resulting signal digitized and sent to a computer. The computer, in turn, does a table lookup according to a previously programmed "frictional characteristic," then converts the corresponding "force" to an analog current that is sent through the string. By virtue of a permanent magnet mounted at the "bowing point," this results in a corresponding physical force exerted on the string. The process is repeated at a sampling rate up to $125 \mathrm{kHz}$.I In the present experiments, the string was $1.40 \mathrm{~m}$ long and had a fundamental frequency near $70 \mathrm{~Hz}$; the magnetic field extended over a length of approximately $2.5 \mathrm{~cm}$, which is to be thought of as the "width" of the equivalent bow; and the frictional characteristic was defined by the function.

$F(v)=F_{n}\left(0 / \varepsilon_{1}\right) /\left[1+\left(w / v_{n}\right)^{2}\right]$

(4)

The parameters in this function, taking account of all the amplifier gains as well as the sensitivities of the converters, had the (approximate) values

$\mathrm{F}_{0}=0.015 \mathrm{~N}, \mathrm{v}_{0}=0.05 \mathrm{~m} / \mathrm{s} .(5)$

The string itself (made of brass) had a linear density of $1.68 \times 10^{-3} \mathrm{~kg} / \mathrm{m}$ corresponding (for this length and fundamental frequency) to a characteristic impedance of $0.329 \mathrm{~kg} / \mathrm{s}$.

Figure 2 presents data obtained in such a situation. The graph shows the velocity of the "bowed" point of the string as a function of time for three placements of that point, corresponding to $\beta=0.333$ (dashed), $\beta$ 
$=0.250$ (dotted), and,$\beta=0.291$ (solid). All approximate an alternation between "sticking" and "slipping," with the "sticking" velocity the same for all three cases and equal to the programmed bow speed. But whereas the observed duty cycle $\gamma$ corresponds roughly to $\beta$ in the first two cases, it is clear thatthis is not so for the third (solid) curve: Even though, is intermediate between the other two, $\gamma$ is smaller than either one.

Figure 3(a)-(c) corresponds, respectively, to the same three cases as Fig. 2; this time, however, we show the displacement of the string as a function of time, observed at two locations: the bowing point itself (top graph), and a point very near the bridge (bottom graph). As we indicated above, the latter can be considered proportional to the slope of the string at the fixed endpoint and thus to the lateral force exerted by the string on the bridge. In Fig. 3(a) and (b) (corresponding to $\beta=1 / 3$ and 1/4), we see the characteristic "staircase" function near the bridge, corresponding to the fact that certain harmonics must be missing, as discussed above. In Fig. 3(c). on the other hand, there is (when observed near the bridge) a marked enhancement of certain harmonics, the effect of which is the narrowing of the duty cycle at the bowing point, in agreement with what our considerations of stability would suggest. This motion is quite similar to the one described by Lawergren;'7 according to McIntyre et al., $\underline{18}$ it is on occasion musically useful as well.

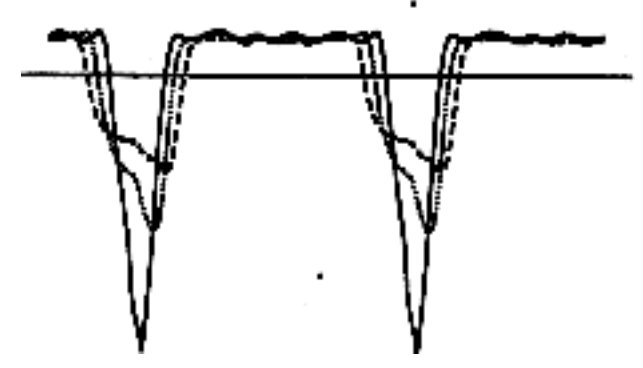

FIG. 2. Velocity of the bowing point as a function of time obtained on the digital bow under three condition: $\beta=0,333$ (dashed), $\beta=0.333$ (doted), and $\beta=0.293$ (solid). The frequency is $70.16 \mathrm{Hzs}$ and two complete periods are shown. The absolute phase is arbitrarily chosen, to superpose the "sliping" pulse of the three cue.

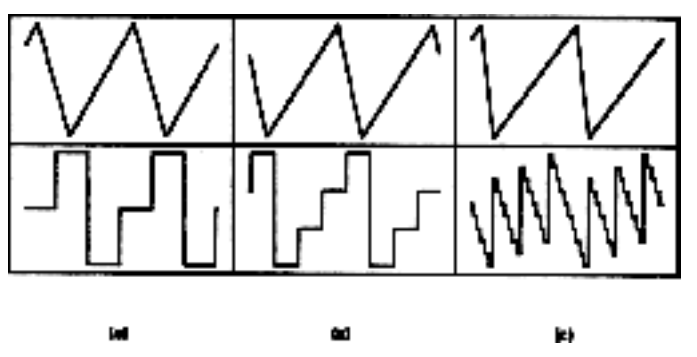

FIG. 3. Displacement of the slring as a function of lime, obtained on the digital bow under the conditions of Fig. (a) $=0.333$. (b) $1=0.250$, (c) $\beta=293$. In each case, the upper curve is taken at the bowing point, the lower one at a point near the bridge. The absolute phases and the vertical scales are arbitrary for each of the six curves.

When the "bow" (that is, the magnet-photodetector assembly) is moved slowly from, say, $\beta=1 / 4$ to a "more irrational" position like $\beta=0.291$, one observes the wave shape changing continuously from that of Fig. 3 (b) to that of 3 (c). In that way, the large quasisinusoidal admixture to the Helmholtz motion seen in the lower graph of 3 (c) joins continuously to the staircase characteristic of 3(b) [or 3(a)], making the latter intuitively more palatable.

\section{Multikink Simulations}

In order to clarify the experimental results of the previous section, we attempted to simulate results such as those in Fig. 3 by constructing low- $Y$ motions as superpositionsof Helmholtz motions according to the 
procedure of Appendix A. Our computer program accepts and $\beta$ as rational fractions $\mathrm{m} / \mathrm{P}$ and $\mathrm{M} / \mathrm{P}$; it then superposes the appropriate number of Helmholtz kinks with the right spacing and produces the displacement as a function of time, either at the bow location $\beta$, or at a point very close to the end of the string. In this way curves are produced that should simulate, respec- tively, the top and bottom graphs in Fig. 3.

Figure 4(a)-(c) shows computed results corresponding to the graphs in Fig. 3. For (a) we took $\mathrm{m}=1, \mathrm{P}=3$, $\mathrm{M}=1$; for (b), $\mathrm{m}=1, \mathrm{P}=4, \mathrm{M}=1$. For (c), $\beta$ was taken to be midway between the other two at $7 / 24$, so that $\mathrm{m}=7, \mathrm{P}=24$; the choice $\mathrm{M}=3$ then produced the closest correspondence between experiment and simulation. In other words, Fig.4(c) is a two-velocity motion at the bowing point whose duty cycle is somewhat less than half of its Helmholtz value, that is, $3 / 24$ compared to $7 / 24$. Naturally, the absolute phase in these simulations is arbitrary but was chosen to resemble as much as possible the data of Fig. 3.

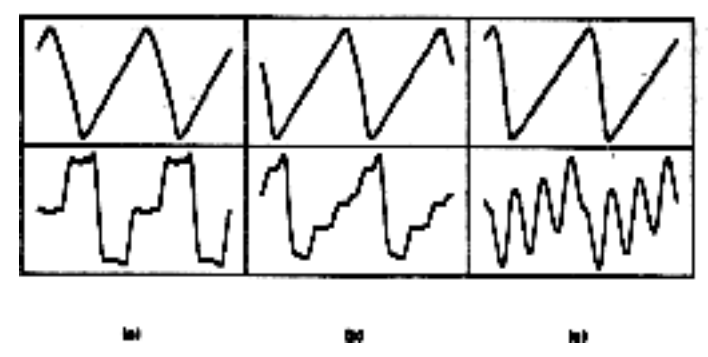

FIG. 4. Simulted displacements at the bowing point (top)and at a point near the bridge (bottom) contructed as superpositions of Helmholtz motions, with conditions (including abslolute phase) chosen to correpond to the data of Fig. 3. (a) $\beta=\gamma=1 / 3$; (b) $\beta=\gamma=1 / 4$; (c) $\beta$ $=7 / 24, \gamma=3 / 24$.

It should be noted that, since $\mathrm{m}=1$ for Fig. 4(a) and (b), no two-velociy motion of lower duty cycle is possible. However, in Fig. (c), $\gamma$ is not as small as it can be. For comparison, Fig. 5(a) shows a pure Helmholtz motion for $\beta=7 / 24$, while 5 (b) illustrates the lowest possible duty cycle, corresponding to $\gamma=$ $1 / 24$ (that is, $M=1$ ) for the same $\beta$. Clearly, the observed motion, as simulated in Fig. 4(c), is narrower than the Helmholtz motion but not as narrow as is kinematically possible.

That there must be a dynamical lower limit onl $\gamma$ for a given $\beta$ is, of course, obvious, since, as $\mathrm{P}$ becomes larger, the purely kinematical limit gets smaller and smaller, becoming zero for irrational values of $\beta$. In attempting to explain this dynamical lower limit, three factors come to mind: the stiffness of the string. the width of the bow, and the presence of energy losses. All three constitute violations of the assumptions that we have made in defining our model.

Although we are not able, at this moment, to treat these complications in detail. We found it heuristically interesting to calculate the energies corresponding to the two-velocity motions of given $\beta$, the idea being that, when dissipation is introduced. a larger stored energy may also correspond to a larger rate of energy dissipation. The larger power requirement from the bow may then make the motion more difficult to maintain.

The energy tored in a simple Helmholtz motion is easily calculated at a sum of kinetic and potential energy densities integrated over the string. If the maximum lateral displacement of the kink (which occurs when it passes the center of the string) is $\mathrm{A}_{0}$, the instantaneous string displacement at time $\mathrm{t}$ is given by where $\mathrm{p}$ is the linear density and $\mathrm{T}=\mathrm{\rho}_{\mathrm{c}} \underline{2}$ is the static tension. 


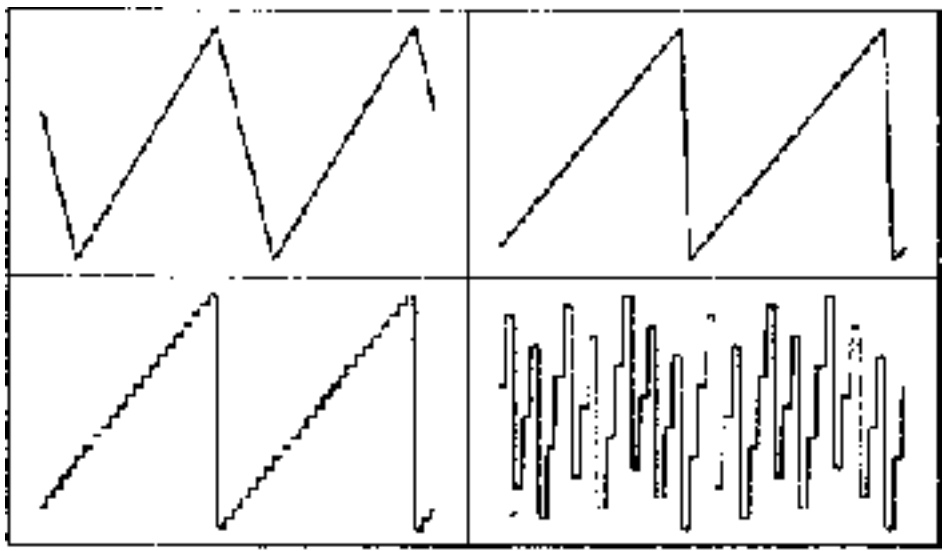

(a.'

(b)

FIG. 5. Simulilated displacement at the bowing point (top) and at the point near the bridge (bottom), constructed as in Fig. 4:(a) for the pure Helholtz motion at $\beta=7 / 24$, (b) for the shortest possible duty cycle at the same $\beta$

$v(x, t)= \begin{cases}4 A_{1} x(L-c) / L^{2}, & \text { for } x<c t, \\ A_{A} A_{0} c t(L-x) / L^{2}, & \text { for } x>c t_{1}\end{cases}$

where $\mathrm{c}$ is the speed of transverse waves. The total energy then becomes

$$
\begin{aligned}
E & =\frac{1}{2} \rho \int_{i 1}^{L}\left(\frac{\partial \psi}{\partial t}\right)^{2} d x+\frac{1}{2} T \int_{0}^{L}\left(\frac{\partial \psi^{\prime}}{\partial x}\right)^{2} d x \\
& =(8 / 3) T A_{1}^{2} / L,
\end{aligned}
$$

When more than one kink is present, the energies cannot, of course. be simply added, since the individual kinks are not orthogonal to each other. Nonetheless, the calculation for any given superposition is straightforward, especially when done by computer.

On the other hand, the two-velocity motions for various $\gamma$ but given, $\beta$ do not have the same lateral displacements; rather, they must (if the comparison is to be meaningful) be normalized to the same value of bow speed $v_{\mathrm{b}}$. Since the construction that determines this depends on the frictional characteristic, as shown in Fig. 1, it cannot be given for a general case. Nonetheless, an excellent approximation is obtained by assuming, for this purpose, that the sticking slope is infinite. In that case, the bow speed is related to $\Delta v$ , the difference in velocities of the two-velocity motion, by

$\mathrm{v}_{\mathrm{b}}=\mathrm{Y}_{\Delta \mathrm{v}}$

allowing the amplitude of the component Helmholtz motion to be determined.

Table I shows the computed results for various two-velocity motions with $\beta=7 / 24=0.291$, the case discussed above, normalized to the simple Helmholtz motion. It is seen that the energy does, indeed, rise steeply as $Y$ is made smaller, making this a possible factor in putting a lower limit on duty cycles actually observed.

TABLE 1. Relative energies of two-velocity motions, normalized to a fixed bow speed, for $\beta=7 / 24$ and varying $\gamma$. The energy for $\gamma=\beta$ is arbitrarily taken as unity. The middle column gives the number of Helmholtz kinks required to construct each motion. 


\begin{tabular}{|c|c|c|}
\hline$y$ & $\begin{array}{l}\text { Number } \\
\text { or kinks }\end{array}$ & $\begin{array}{l}\text { Relative } \\
\text { energy }\end{array}$ \\
\hline $1 / 24$ & 7 & 49.00 \\
\hline $2 / 24$ & 14 & 18.577 \\
\hline $3 / 24$ & 21 & 6.050 \\
\hline $4 / 24$ & 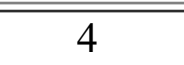 & 4.261 \\
\hline $5 / 24$ & 11 & 3.923 \\
\hline $6 / 24$ & 18 & 2.216 \\
\hline $7 / 24$ & 1 & 1.000 \\
\hline $8 / 24$ & 8 & 1.449 \\
\hline 9/24 & 15 & 1.278 \\
\hline $10 / 24$ & 22 & 0.743 \\
\hline $11 / 24$ & 5 & 0.811 \\
\hline $12 / 24$ & 12 & 0.852 \\
\hline $13 / 24$ & 19 & 0.580 \\
\hline $14 / 24$ & 2 & 0.379 \\
\hline $15 / 24$ & 9 & $\begin{array}{l}0.460 \\
\end{array}$ \\
\hline $16 / 24$ & 16 & 0.362 \\
\hline $17 / 24$ & 23 & 0.170 \\
\hline $18 / 24$ & 6 & 0.246 \\
\hline $19 / 24$ & 13 & 0.272 \\
\hline $20 / 24$ & 20 & 0.170 \\
\hline $21 / 24$ & 3 & 0.123 \\
\hline $22 / 24$ & 10 & 0.154 \\
\hline $23 / 24$ & 17 & 0.093 \\
\hline
\end{tabular}

\section{Instability Simulations}

As we mentioned in Sec. IV, the otherwise plausible idea that the (positive) sticking resistance $\mathrm{R}_{\mathrm{a}}$, and the (negative) slipping resistance $\mathrm{R}_{\mathrm{g}}$ can somehow compensate each other in the determination of a stability criterion is not correct, since there exist possible perturbation patterns ("between- the-raindrops" motions, Appendix B) that can synchronize their appearance at the bowing point to coincide with the slipping parts of the cycle. To explore this phenomenon, we constructed a computer program to simulate perturbation of the Helmholtz motion in the linear regime, that is, when the perturbation is small compared to the Helmholtz motion itself. This is done by describing the string by an array along which the two waves-one moving to the right, the other to the left-propagate without change, except that at the point $\beta_{\mathrm{L}}$ they are scattered by a resistance $R$ which has the value $R_{g}$, for a fraction $\beta$ of the period and the value $R_{a}$ for the remaining fraction $1-\beta$. For this purpose, we use the elementry formulas that give the reflection coefficient at such a point as $-\mathrm{R} /(2+\mathrm{R})$, and the corresponding transmission coefficient a $2 /(2+\mathrm{R})$, where $\mathrm{R}$ is, of course, measured in units of tht characteristic impedance of the string. We used a "string" of points, with $\beta$ $=7 / 23$ and perfect reflection at the endoint. The initial form of the perturbation was taken, quite arbitrarily, as sinusoidal, correponding to the fundamental of a free string (that is one without a bow); other choices of initial conditions support the same conclusions.

Figure 6 shows some computed results. Each of the four graphs give the displacement of the point nearest 
one end of the string, plotted at the initial time and then once per period (that is, every 46 time increments) for a total time of 100 periods; they differ, however, in the values assigned to the sticking and slipping resistances.

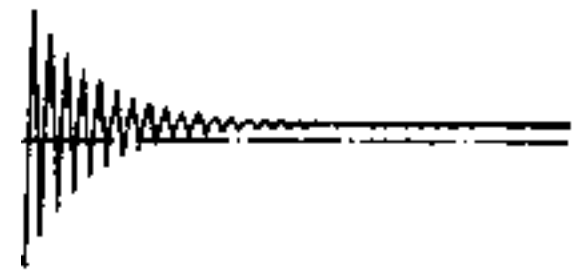

(a)

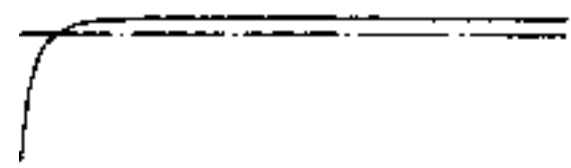

(b)

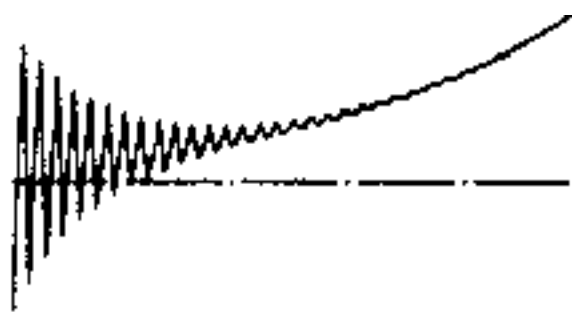

(c)

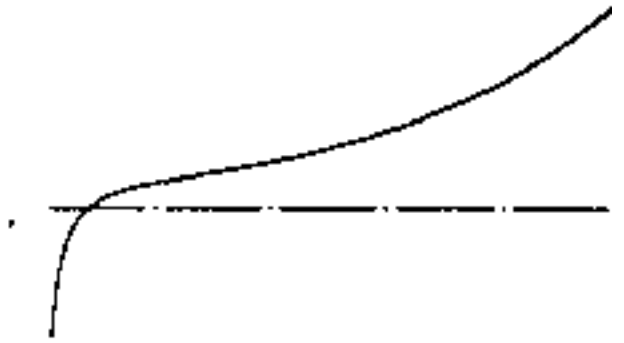

(d)
FIG. 6 Perturbations computed in th linear regime for a "string" of 23 points, with $\beta=7 / 23$, for various value of the sticking and slipping resistances. The displacement of the point nearest one end is plotted once per period, for a total interval of 100 periods:
(a) $\mathrm{R}_{\mathrm{a}}=50, \mathrm{R}_{\mathrm{g}}=\mathrm{O}$;
(b) $\mathrm{R}_{\mathrm{a}}=0.5, \mathrm{R}_{\mathrm{g}}=\mathrm{O}$;
(c) $\mathrm{R}_{\mathrm{a}}=50, \mathrm{R}_{\mathrm{g}}=-0.05$;
(d) $R_{a}=0.5, R_{g}=-0.05$.

In Fig. 6(a) and (b), the slipping resistance is taken to be $\mathrm{R}_{\mathrm{g}}=0$, whereas the sticking resistance (expressed in units of the characteristic impedance of the string) is $R_{a}=50$ in (a) and $R_{a}=0.5$ in (b). The first shows a damped subharmonic that repeats every three periods; this is consistent with the statement made by Mclntyre et al..$\underline{14}$ that the possible subharmonic orders are the two integers closest to $1 / 3$, which, in our case, is $23 / 7=3.29$. The damping is, presumably, due to the finiteness of $R_{a}$; we note, however, that as this perturbation dies away, the value asymptotically approached in the graph is not zero. The existence of this asymptote indicates another, undamped, perturbation which has the same values at times that are separated by one period; it takes place, in other words, at the fundamental frequency of the string. This is, of course, the type of perturbation we mentioned earlier and described as a "between- the-raindrops" motion, a conclusion that is supported by examining the shape of the perturbation at the end of the simulation computation.

In Fig. 6 (b) the slipping resistance is kept at zero but the sticking resistance is lowered to $R_{a}=0.5$. The subharmonic is now so strongly damped as to be essentially unrecognizable, yet the nonzero asymptotic value is not only still there but entirely unchanged in magnitude. as is to be epected from a perturbation that never sees the sticking part of the bow cycle at all.

Finally, in Fig. 6(c) and (d), the computation is repeated after setting the slipping resistance to the mildly negative value $R_{g}=-0.05$; in each figure, $R_{a}$ is the same as in the graph immediately to its left. It is clear that the previously constant asymptotic perturbation now becomes a growing one, since it is absent from the bow position when the bow sticks but present when it slips. The massive damping caused by $R_{a}$ in Fig. 6(d) has no appreciable effect on this growth rate, even though the amplification is quite small compared to this damping, and the duration of the slipping cycle is shorter. 


\section{Discussion}

Effects connected with the narrowing of the duty cycle of bowed-string motion have also been observed with physical bows by Lazarus $\frac{19}{}$ and Lawergren. $\frac{17}{}$ The latter, in particular, interprets his observations in terms of a superposition of a pure Helmholtz motion and a single sinusoid of noninteger period which has nodes at the string endpoints and a slope discontinuity at the observation point. No theoretical reason is, however, given by Lawergren why this particular function should be preferred. In fact, Appendix A proves that, at an arbitrary location on an ideal string (one for which, is not rational), there is no limit to the narrowing of the duty cycle that is kinematically possible, and the exact nature of the observed dynamical limit is not yet understood.

It may be worth, in this connection, to mention another phenomenon observed with the digital bow by Chayé ${ }^{20}$; for the case in which is a ratio of small integers but the numerator is not unity. She found that, if $\beta=\mathrm{m} / \mathrm{P}$, the duty cycle $\gamma$ of the motion at the bowing point tends always to be $1 / \mathrm{P}$. For example, for $\beta=$ $1 / 7,2 / 7,3 / 7,4 / 7,5 / 7$, or $6 / 7, \gamma$ is always $1 / 7$. In other words, in this case it is, indeed, the shortest $\gamma$ that is chosen, consistent with the idea that, due to stability considerations, the motion which is preferred is the one that minimizes the time spent in the negative-resistance region of the characteristic. It is not clear to what degree this narrow- ing can be avoided on real violins by "correct" bowing; comparison with the digital bow is complicated by its relatively large width, as well as the fact that the magnitude of its force is limited by effects of string heating.

Our discussion of Sec. IV, along with the computer simulations of Sec. VII, indicates that the apparent experimental stability of Helmholtz motion remains a puzzle, in that (unlike what has been previously believed) this stability cannot be explained by changing the "effective" sticking resistance from an infinite to a finite value. As far as we can see, the truth must lie in one or more of the following three possibilities: (a) The stability comes from some other source of dissipation (such as losses at the supports); (b) the stability comes from other deviations from the "perfect" model, such as bow width. string stiffness, or perhaps bow dynamics: the latter would make it impossible to represent the frictional characteristic a an instantaneous. time-independent function: and (c) the Helmholtz motion is, in fact, not stable but its instabilities themselves stabilize at fairly small amplitudes so that casual experiments appear to suport the notion that Helmholtz motion is being executed. It should be noted in this connection that the simulations of Sec. Vll were computed in a linear regime and thus throw no light on what happens to the perturbations after they have begun to grow.

\section{Acknowledgments}

We are grateful to M. Mclntyre and to J. Woodhouse for extensive discussions which not only improved this paper but also taught us a lot of good physics. We wish also to acknowledge the helpful comments of many other colleagues, among them the late Professor L. Cremer, X. Boutillon, and R. Schumacher; and to express our awestruck appreciation to Bennett Smith, who built and interfaced the digital processing equipment used for the digital bow.

\section{Appendix A: Possible two-velocity Motions}

We wish to prove the following theorem: If a free string moves in such a way that at location $\beta_{\mathrm{L}}$, where $\beta_{=}$ $\mathrm{m} / \mathrm{P}$ and the fraction is in its lowest terms, the motion consists of two velocities that alternate in the course of a period, the possible values of the duty cycle $\gamma$ are $\mathrm{M} / \mathrm{P}$, where $\mathrm{M}=1,2, \ldots, \mathrm{P}-1$. This result is clearly present in the work of Raman, but we find the following proof rather more accessible.

We begin by noting that $\beta=\mathrm{m} / \mathrm{P}$ is a node of all harmonics whose order is a multiple of $\mathrm{P}$, so that the Fourier spectrum of the velocity observed at that point must lack those harmonics. To impose this condition, we first calculate the amplitude of the nth harmonic of a square pulse of duty cycle $\gamma$; apart from 
an unimportant normalizing factor, it is given by

$$
\mathrm{A}_{\mathrm{n}}(\gamma)=[1-\exp (2 \pi \mathrm{in} \gamma)] / \mathrm{n}
$$

By setting $A_{n}(y)=O$, we obtain

$$
\mathrm{P}_{\gamma}=\text { integer. }
$$

Thus the Pth harmonic will vanish if and only if $\gamma$ has the form $\mathrm{M} / \mathrm{P}$, where $\mathrm{M}$ is an integer. We note also that the vanishing of the Pth harmonic automatically guarantees the vanishing of all its multiples. as required.

If $\gamma$ does have the above form, we know from the discussion of Sec. II that a corresponding two-velocity motion must exist. It may, nonetheless, be useful to facilitate its visualization by giving an explicit construction for it as a superposition of simple Helmholtz motions as advocated by Kubota. $\underline{21-22}$

The construction is as follows. Consider the multiples of m modulo P. If one such is equal to zero, it means that a certain multiple of $\mathrm{m}$ is equal to a certain multiple of $\mathrm{P}$; and, since $\mathrm{m}$ and $\mathrm{P}$ have (by assumption) no common factors, it follows that the number by which $\mathrm{m}$ is multiplied to produce zero (modulo $\mathrm{P}$ ) must itself be a multiple of $\mathrm{P}$. In other words, if $\mathrm{m}$ is multiplied successively by $0,1, \ldots, \mathrm{P}-1$, all of which are less than $\mathrm{P}$, no two results can he equal (modulo $\mathrm{P}$ ). On the other hand, there are exactly $\mathrm{P}$ numbers in the range of all integers modulo $\mathrm{P}$, so that each of them must appear once and only once in the set of posible results of multiplying $\mathrm{m}$ by some integer and reducing the result modulo $\mathrm{P}$. Let $\mathrm{M}$ be all integer in the set $1,2, \ldots, \mathrm{P}-1$. and $\mathrm{N}$ the integer such that $\mathrm{Nm} \bmod \mathrm{P}=\mathrm{M}$.

Consider now $\mathrm{N}$ simple Helmholtz motions, all of the same amplitude, coexisting on one string, and spaced apart by a fraction $\mathrm{m} / \mathrm{P}$ of a period. In terms of a spatial distribution, it is convenient to viualize the string as an endless conveyor belt looped around a pair of pulleys located at the endpoints of the string, and moving with the speed of transverse waves on the string; half of the belt can then be thought of as carrying waves traveling to the right, the other half to the left. The N Helmholtz motions under consideration can then be represented as marks on the conveyor belt indicating the location of the kinks. Note that the total length of the belt is $2 \mathrm{~L}$, so that the spatial interval between successive kinks is $2(\mathrm{~m} / \mathrm{P}) \mathrm{L}$.

Consider now the location $(\mathrm{m} / \mathrm{P}) \mathrm{L}$ along the string as measured from one end which, for convenience, we shall call the "bridge." Because the kinks are spaced apart by twice that distance, it follows that, whenever a kink passes this observation point moving toward the bridge, another will be passing it moving in the opposite direction. Since the velocity transition of a Helmholtz motion occurs whenever the kink passes the observation point, we see that in our present case each such transition will be canceled by an equal and opposite one. The two exceptions are when the first kink of the sequence passes in the direction toward the bridge, and when the last one passes in the other direction. Since there are N kinks, spaced apart by $\mathrm{m} / \mathrm{P}$ of a period, the time interval between the first and the last, figured as a fraction of a period, is $\mathrm{Nm} / \mathrm{P}$. In order to reduce it to a propr fraction, we reduce the numerator modulo $\mathrm{P}$; and since $\mathrm{Nm} \bmod \mathrm{P}=\mathrm{M}$ by construction, we see that the fraction of a period that elapes between the two transitions that do occur exactly $\mathrm{M} / \mathrm{P}$, as was required.

For the "multikink simulations" of Sec. VI, we used the above construction but modified it further by complementing each Helmholtz kink with Pnegative "subsidiary" kinks in order to produce the "staircase" behavior explained in Sec. III. This modification does not, of course, affect the motion observed at $\beta=\mathrm{m} / \mathrm{P}$ and is hence irrelevant for the theorem stated at the beginning of this Appendix. It is, however, important for describing the motion at other points and for the calculation of energies. 


\section{Appendix B: "between-the-Raindrops" Motions}

The conveyor-belt picture of Appendix A is useful also for illustrating the existence of "between-theraindrops" motions, that is, possible motions of the free string for which the displacement at the bow position is identically zero except for a fraction $\beta$ of the cycle (which we associate with the slipping time of the bow). Now it is clear that, if $\beta=\mathrm{m} / \mathrm{P}$, a rational number, then normal modes whose orders are multiples of $\mathrm{P}$ will have nodes at the bow position and thus give zero displacement at the bow at all times. We will therefore assume that $\beta$ is not rational, in order to avoid being confused by the existence of those modes.

Let our conveyor belt, whose total (unfolded) length is $2 \mathrm{~L}$, have associated with it a displacement pattern that is periodic with period $2^{\beta} \mathrm{L}$; since $\beta$ is, by assumption, not rational, it is clear that somewhere there will be a junction point where this periodicity is broken. If we now imagine the belt mounted on the pulleys (of which one represents the "bridge") and put in motion with the correct wave velocity, the equations of the free string will be satisfied, in that the resulting displacement will contain a wave going to the right and another going to the left, each of which returns from its respective endpoint reflected with a change of sign (since the "top" of the conveyor belt going to the right turns into the "bottom" going to the left ).

Consider now the "bow position" $\beta_{\mathrm{L}}$. Since the two points of the belt passing it at a given moment are separated by a distance $2^{\beta_{L}}$ (measured around the "bridge" pulley), and since the pattern is, by construction, periodic with that quantity as period, the displacements corresponding to the left-going and right-going waves will, at each moment, be equal and opposite, resulting in a total displacement at the bow position which vanishes identically. This condition will not hold, however, when the above-mentioned junction point is passing from the "bow position" to the "bridge" and back again; and since this distance is a fraction, $\beta$ of the total belt length, the time for which the displacement is not zero will, be a fraction of the period. Thus the existence of between-the-raindrops" motions is proved.

It is worth noting that motions of even shorter duration at the bow position can be constructed by choosing displacement patterns whose spatial period along the belt is an integer submultiple of $2 \beta_{\mathrm{L}}$. In fact, it is easy to convince oneself (in analogy with the construction of short duty cycles as described in Appendix A) that, for the case when $\beta$ is irrational, motions exist which vanish at the bow position for all but an arbitrarily small fraction of the period.

\section{Notes}

H. v. Helmholtz, On the Sensations Of tone (Dover. New York, 1954) (English translation of the German edition of 1877).

L. Cremer, The Physics of the Violin (MIT, Cambridge, MA, 1984) (English translation of the

German edition of 1981).

R. T. Schumacher, "Self-sustained oscillations of the bowed string, "Acustica 43, 109-120 (1979).

M. E. Mclntyre and J. Woodhouse, "On the fundamentals of bowed-string dynamics," Acustica 43, 93-108 (1979).

C. V. Raman, "On the mechanical theory of the vibrations of bowed strings and of musical instruments of the violin family, with experimental verification of the results: Part I," Indian Assoc. Cult. Sci. Bul. 15,1-158 (1918). 
M. E. Mclntyre (private communication).

$[7]$

M. E. Mclntyre, R. T. Schumacher, and J. Woodhouse, On the oscillations of musical instruments," J. Acoust. Soc Am. 74, 1325-1345 (1983).

[8]

See Ref. 2, Chap. 6.

[9]

See Ref. 2, Sec. 3.6.

[10]

[11]

See Ref. 1, p. 84

See Ref. 2, p. 51.

[12]

F. G. Friedlander. "On the oscillations of the bowed string," Proc. Cambridge Philos. Soc. 49, 516530 (1953).

M. E. Mclntyre, R. T. Schumacher. and J. Woodhouse, "Aperiodicity in bowed-string motion," Acustica 49, 13-32 (1981).

See Ref. 2, p. 43.

[15]

J. C. Schelleng, "The bowed string and the player," J. Acoust. Soc. Am. 53, 26-41 (1973) .

R. Caussé and G. Weinreich, "Simulation and further experiments with the digital bow," 13th International Congress on Acoustics, Belgrade Vol. 3, p. 83 (1989).

B. Lawergren, "On the motion of bowed violin strings," Acustica 44, 194-206 (1980).

[19]

See Ref. 7, p. 1335.

H. Lazarus, "Die Behandlung der selbsterregten Kippschwingungen der gestrichenen Saite mit Hilfe der endlichen Laplacetransformation," thesis, Technical University of Berlin (1973).

I. Chayé, "Etude de l'interaction entre la corde et l'archet," Memoire de D.E.A., Université de Technologie de Compiègne (1987).

[21]

H. Kubota and M. Kondo, "Tables of numbers of the Helmholtzian waves on a bowed string," 11th ICA, Paris, Vol. 4, p. 367 (1983).

[22]

H. Kubota, "Velocity measurement for the bowed string and plural Helmholtz waves," Catgut Acoustical Society International Symposium, Hartford (1986). 